\title{
Nuclear magnetic relaxation in liquid ${ }^{3} \mathrm{He}$ and ${ }^{3} \mathrm{He}-{ }^{4} \mathrm{He}$ mixtures
}

\author{
A. S. van Steenbergen and S. A. J. Wiegers \\ Research Institute for Materials, High Field Magnet Laboratory, University of Nijmegen, NL-6525 ED Nijmegen, The Netherlands \\ P. E. Wolf \\ Centre de Recherche sur les Très Basses Températures, CNRS, BP 166, F-39042 Grenoble Cédex 9, France \\ J. A. A. J. Perenboom and J. C. Maan \\ Research Institute for Materials, High Field Magnet Laboratory, University of Nijmegen, NL-6525 ED Nijmegen, The Netherlands
}

(Received 17 February 1998)

\begin{abstract}
The spin-lattice relaxation time $T_{1}$ of liquid ${ }^{3} \mathrm{He}$ and ${ }^{3} \mathrm{He}-{ }^{4} \mathrm{He}$ mixtures is determined by two parallel relaxation processes: intrinsic relaxation, which is caused by dipolar interaction between the ${ }^{3} \mathrm{He}$ nuclear spins, and surface relaxation, due to interaction of the ${ }^{3} \mathrm{He}$ nuclear spins with the magnetic moments at the walls of the experimental cell. Using a type of torque magnetometer, we have measured $T_{1}$ of liquid ${ }^{3} \mathrm{He}$ containing $0.5 \%{ }^{4} \mathrm{He}$ and ${ }^{3} \mathrm{He}-{ }^{4} \mathrm{He}$ mixtures with a ${ }^{3} \mathrm{He}$ concentration ranging from 6 to $95 \%$, as a function of magnetic field up to $22 \mathrm{~T}$ at temperatures between $40 \mathrm{mK}$ and $1 \mathrm{~K}$. Due to the difference in their magnetic-field dependences, we have been able to separate the intrinsic and surface contributions to $T_{1}$. Our measurements reveal a surface relaxation mechanism for liquid ${ }^{3} \mathrm{He}$, with a relaxation time proportional to the square of the magnetic field, which can be described by the classical relaxation theory of Bloembergen, Purcell, and Pound. We relate the observed classical relaxation mechanism to the dynamics of the ${ }^{3} \mathrm{He}$ atoms in the ${ }^{4} \mathrm{He}$ film at the surface. The temperature dependence of the surface relaxation time $T_{\mathrm{s}}$ is consistent with the hypothesis that the surface relaxation is caused by diffusive motion of ${ }^{3} \mathrm{He}$ atoms near the surface. This mechanism would naturally explain the previously unexplained observations that $T_{\mathrm{s}}$ is inversely proportional to the diffusion coefficient, while $T_{\mathrm{s}}$ is clearly larger than the diffusion time. We find the intrinsic relaxation time $T_{\text {in }}$ of the pure liquid ${ }^{3} \mathrm{He}$ in good agreement with existing Fermi-liquid theory, and observe the $T_{\text {in }}$ of the ${ }^{3} \mathrm{He}-{ }^{4} \mathrm{He}$ mixtures at $1 \mathrm{~K}$ to be proportional to the ${ }^{3} \mathrm{He}$ concentration, in agreement with theoretical predictions.
\end{abstract}

[S0163-1829(98)07025-8]

\section{INTRODUCTION}

Liquid ${ }^{3} \mathrm{He}$ is a thoroughly studied model system for correlated fermions and for nuclear magnetic relaxation phenomena in a monoatomic liquid down to the lowest temperatures. Because of its simple atomic structure, intra-atomic relaxation processes are absent, and dipolar interactions between the ${ }^{3} \mathrm{He}$ nuclear spins determine the intrinsic relaxation time $T_{\text {in }}$. Hence, measurement of $T_{\text {in }}$ probes the dynamics in the liquid. However, like in all magnetic liquids and gases, the spin-lattice relaxation in liquid ${ }^{3} \mathrm{He}$ and ${ }^{3} \mathrm{He}-{ }^{4} \mathrm{He}$ mixtures observed in an experiment is the combined result of intrinsic relaxation and surface relaxation at the unavoidable surfaces of the experimental cell. Therefore, $T_{\text {in }}$ as a function of temperature cannot be determined unambiguously from $T_{1}$, as both the surface relaxation time $T_{\mathrm{s}}$ and $T_{\text {in }}$ are a priori unknown and temperature dependent. ${ }^{1-4}$ In the so-called diffusion limited regime, the surface relaxation time is dominated by the time the ${ }^{3} \mathrm{He}$ atoms need to reach the wall, and decreases approximately with $T^{2}$ at low temperatures. Therefore, surface relaxation can be fast, and the determination of $T_{\text {in }}$ is difficult at very low temperatures. Consequently, although $T_{\text {in }}$ of liquid ${ }^{3} \mathrm{He}$ is expected to increase approximately as $T^{-2}$ in the Fermi-liquid regime ${ }^{5-7}$ (below $100 \mathrm{mK}$ for liquid ${ }^{3} \mathrm{He}$ ), this behavior has never been directly observed due to the presence of surface relaxation.

We have been able to determine $T_{\text {in }}$ of liquid ${ }^{3} \mathrm{He}$ and ${ }^{3} \mathrm{He}-{ }^{4} \mathrm{He}$ mixtures in an independent way, using a torque magnetometer technique. ${ }^{8}$ In this paper we present an extensive experimental study on $T_{1}$ of liquid ${ }^{3} \mathrm{He}$ containing $0.5 \%$ ${ }^{4} \mathrm{He}$ and ${ }^{3} \mathrm{He}-{ }^{4} \mathrm{He}$ mixtures with a ${ }^{3} \mathrm{He}$ concentration ranging from 6 to $95 \%$, as a function of magnetic field up to $22 \mathrm{~T}$ at temperatures between $40 \mathrm{mK}$ and $1 \mathrm{~K}$. Our data allow unambiguous determination of $T_{\text {in }}$ of liquid ${ }^{3} \mathrm{He}$ and ${ }^{3} \mathrm{He}-{ }^{4} \mathrm{He}$ mixtures as a function of temperature and ${ }^{3} \mathrm{He}$ concentration, and a detailed study of the surface relaxation time $T_{\mathrm{s}}$.

We have chosen to study ${ }^{3} \mathrm{He}$ containing ${ }^{4} \mathrm{He}$, since in this system the surface is covered with a thin layer of ${ }^{4} \mathrm{He}$ (even if the ${ }^{4} \mathrm{He}$ concentration is as low as $0.5 \%$ ), which leads to much longer relaxation times than in the case of pure ${ }^{3} \mathrm{He},{ }^{3}$ where a solid ${ }^{3} \mathrm{He}$ layer is formed at the surface. For a solid layer coverage, a linear temperature and magnetic-field dependence of $T_{1}$ has been found. ${ }^{9}$ Furthermore, the dependence of the intrinsic relaxation time $T_{\text {in }}$ on the ${ }^{3} \mathrm{He}$ density can be observed over a wide range by varying the ${ }^{3} \mathrm{He}$ concentration in the ${ }^{3} \mathrm{He}^{4} \mathrm{He}$ mixtures. Therefore, intrinsic relaxation of liquid ${ }^{3} \mathrm{He}$ is best studied in ${ }^{3} \mathrm{He}$ systems containing ${ }^{4} \mathrm{He}$. Moreover, relaxation of ${ }^{3} \mathrm{He}$ in ${ }^{4} \mathrm{He}$ plays an essential role in most of the experiments on highly spinpolarized ${ }^{3} \mathrm{He} .{ }^{10,11} \mathrm{~A}$ better understanding of the relaxation processes is necessary to improve the novel techniques for the production of highly spin-polarized ${ }^{3} \mathrm{He} .{ }^{11}$

In our experiments, $T_{1}$ is determined from the exponential decay of the nuclear magnetization, measured with a torsional magnetometer, after a rapid change of the magnetic 


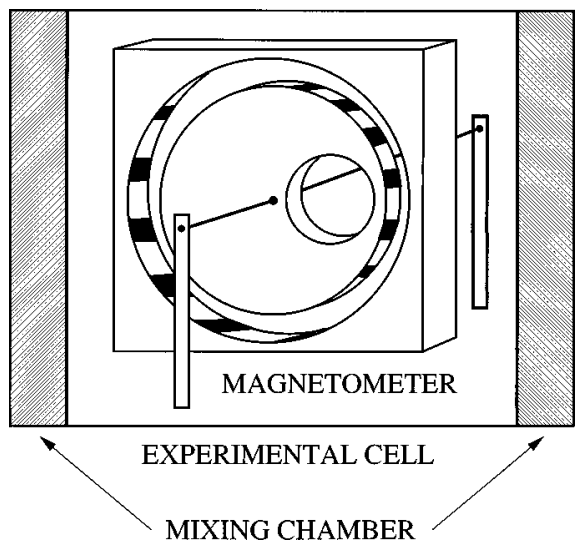

FIG. 1. Schematic drawing of the torsional magnetometer for the measurement of the spin-lattice-relaxation time $T_{1}$ of liquid ${ }^{3} \mathrm{He}$ or ${ }^{3} \mathrm{He}-{ }^{4} \mathrm{He}$ mixtures. The magnetometer is located in the experimental cell, separated from the surrounding mixing chamber (dashed) by a Kapton foil.

field. The advantage of this technique is that it can be used at arbitrary (nonzero) magnetic field. From the measured $T_{1}$ as a function of the magnetic field, the intrinsic and surface contributions to $T_{1}$ can be separated as a result of their different magnetic-field dependences.

The values and temperature dependences of $T_{\text {in }}$ of liquid ${ }^{3} \mathrm{He}$ determined from our experiments are in good agreement with Fermi-liquid theory. ${ }^{5}$ Well above the Fermi temperature at $1 \mathrm{~K}$, we find $T_{\text {in }}$ of the ${ }^{3} \mathrm{He}-{ }^{4} \mathrm{He}$ mixtures to be proportional to the ${ }^{3} \mathrm{He}$ density, in agreement with theoretical predictions. $^{4}$

We have discovered that the surface relaxation time of ${ }^{3} \mathrm{He}$ in the presence of ${ }^{4} \mathrm{He}$ is proportional to the square of the magnetic field, ${ }^{8}$ markedly different from the linear magnetic-field dependence observed in pure ${ }^{3} \mathrm{He} .{ }^{9}$ This behavior has not previously been observed in a Fermi liquid and can surprisingly be described by the theory of Bloembergen, Purcell, and Pound ${ }^{12}$ for nuclear magnetic relaxation in classical fluids, where the nuclear spin is subject to a randomly fluctuating perturbing magnetic field. We relate the observed relaxation to the dynamics of the ${ }^{3} \mathrm{He}$ atoms in the ${ }^{4} \mathrm{He}$ film at the surfaces, where the ${ }^{3} \mathrm{He}$ spin relaxes due to interaction with the inhomogeneous magnetic field of the surface. The exact nature of this interaction however, is still not completely elucidated.

This paper is organized as follows. First we will describe the experimental setup and the procedure to determine $T_{1}$ of liquid ${ }^{3} \mathrm{He}$ and ${ }^{3} \mathrm{He}-{ }^{4} \mathrm{He}$ mixtures. In Sec. III we will present the experimental results, which will be discussed in Sec. IV. A model for the surface relaxation will be presented and the intrinsic relaxation time will be compared to existing Fermiliquid theories. In Sec. V we will summarize the main conclusions.

\section{EXPERIMENTAL SETUP}

The magnetometer, schematically shown in Fig. 1, is made of plastic $\left(\mathrm{Hysol}^{13}\right)$ and consists of a wheel $(15.5 \mathrm{~mm}$ diameter, $10 \mathrm{~mm}$ thick) in a cylindrical housing $(16 \mathrm{~mm}$ inner diameter). ${ }^{14}$ Two holes of equal volume $\left(0.25 \mathrm{~cm}^{3}\right)$ are drilled in the wheel. One of them is closed and empty, the other is open to the liquid (5.6 mm diameter). A torsion wire (manganin, $100 \mu \mathrm{m}$ diameter), glued in the axis of the wheel and stretched between two supports, keeps the wheel concentric in the housing. The rotation of the wheel is measured with capacitors which are formed by electrodes on the outside of the wheel and the inside of the housing (drawn as black squares in Fig. 1). A small coil is wound around the wheel, which can be used for calibration of the signal by passing a current through the coil in a magnetic field.

The magnetometer is mounted in an experimental cell filled with liquid ${ }^{3} \mathrm{He}$ (containing $0.5 \%{ }^{4} \mathrm{He}$ ) or ${ }^{3} \mathrm{He}-{ }^{4} \mathrm{He}$ mixture. We have coated the surface with polystyrene, by dipping all parts of the magnetometer and the cell in a polystyrene/toluene solution, thus reducing the surface relaxation by smoothening the cell surface and reducing its effective area. The cell is placed in the mixing chamber of a homemade plastic dilution refrigerator. ${ }^{15}$ The wall of the cell is a $200 \mu \mathrm{m}$ thick Kapton foil ${ }^{16}$ which acts as heat exchanger between the cell and the surrounding mixing chamber.

The temperature was monitored by a $1 \mathrm{k} \Omega \mathrm{RuO}_{2}$ chip resistor, ${ }^{17}$ which was calibrated against a commercial $\mathrm{Ge}$ thermometer ${ }^{18}$ and a CMN thermometer. As there were no metal parts in the mixing chamber and the experimental cell, apart from the silver electrodes and leads to the magnetometer and thermometers, little eddy-current heating was induced by sweeping the magnetic field, resulting in a total accuracy and stability of the temperature to better than $10 \%$.

By placing the magnetometer somewhat off the center of the magnetic field in a field gradient, the magnetized ${ }^{3} \mathrm{He}$ in the hole experiences a force, and the unbalanced torque causes a small rotation of the wheel. The rotation is capacitively detected in an ac bridge circuit using a ratio transformer ${ }^{19}$ which allows us to measure the magnetization with a resolution of $5 \times 10^{-12} \mathrm{~J} / \mathrm{T}$ at $10 \mathrm{~T}$, corresponding to $10^{-4}$ of the saturation magnetization of the liquid ${ }^{3} \mathrm{He}$ in the hole of the wheel. $T_{1}$ is determined from the exponential decay of the nuclear magnetization after a quick step (much shorter than $T_{1}$ ) of the magnetic field. A typical relaxation curve is shown in Fig. 2.

The main advantage of this nonresonant torque magnetometer is that it can be used at any magnetic field, temperature, and pressure, contrary to, for example, nuclear magnetic resonance, which has to be tuned at every magnetic field. Moreover, the signal of the magnetometer is proportional to the change in magnetization times the field gradient. Because the magnetic-field gradient itself is proportional to the magnetic field, the sensitivity of the magnetometer increases linearly with the magnetic field.

As the magnetometer measures all magnetization, the nuclear magnetization which relaxes during the field sweep is masked by the diamagnetic signal of the ${ }^{3} \mathrm{He}$ and ${ }^{4} \mathrm{He}$ and of the magnetometer itself (background). Therefore, the nuclear paramagnetic relaxation is measured at stable magnetic field, after a rapid sweep of the magnetic field when the diamagnetic contribution to the signal is constant. As it takes at least $10 \mathrm{~s}$ for the magnetometer to stabilize from the diamagnetic signal, the magnetometer cannot measure relaxation times shorter than this stabilization time. 


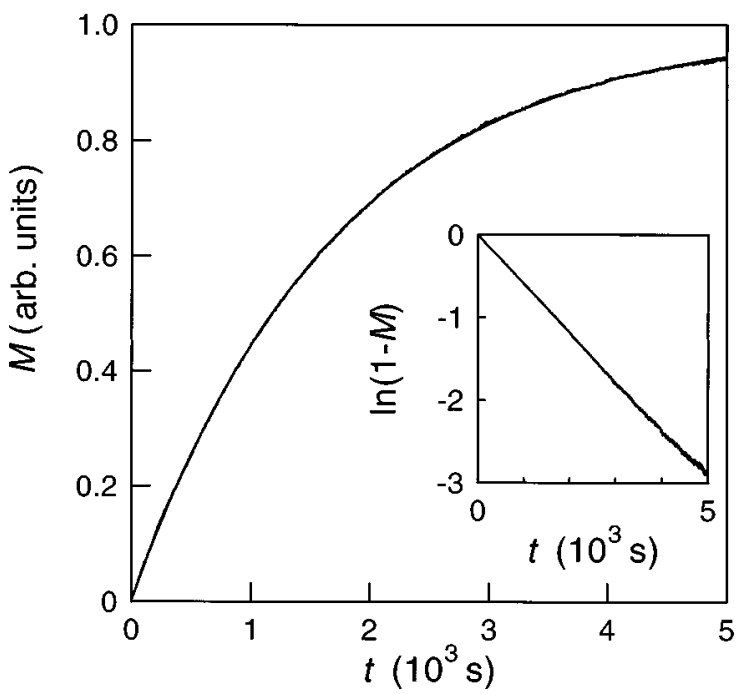

FIG. 2. Relaxation of the nuclear magnetization of liquid ${ }^{3} \mathrm{He}$ containing $0.5 \%{ }^{4} \mathrm{He}$ (at about $80 \mathrm{mK}$ ) after a field sweep from 9 to $13 \mathrm{~T}$ in $40 \mathrm{~s}$ in a Bitter magnet. The exponential fit (with $T_{1}$ $\approx 1700 \mathrm{~s}$ ) is hardly distinguishable from the experimental data. The inset shows the logarithm of the magnetization.

\section{RESULTS}

The relaxation times of saturated vapor-pressure liquid ${ }^{3} \mathrm{He}$ containing $0.5 \%{ }^{4} \mathrm{He}$ are presented in Fig. 3 as a function of the magnetic field at temperatures ranging from 40 to $750 \mathrm{mK} . T_{1}$ shows a gradual change from a low-field region, where it has a complex temperature dependence and increases with magnetic field, to a high-field region, where it approaches a magnetic-field-independent saturation value, which increases with decreasing temperature. Because the ${ }^{3} \mathrm{He}$ nuclear polarization remains below 5\% even for the largest fields studied, we do not expect any modification of the intrinsic (bulk) relaxation due to the magnetic field. ${ }^{20}$ The

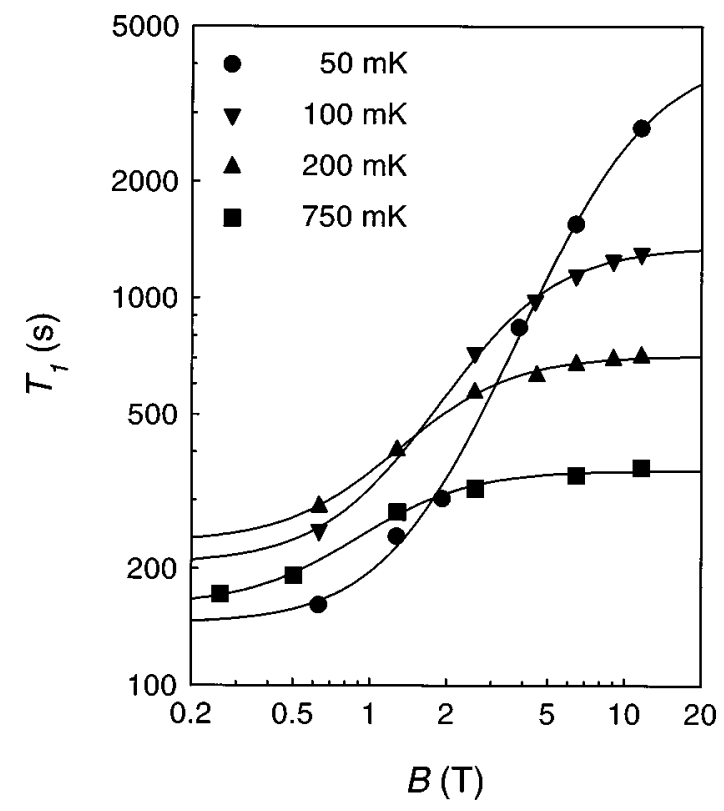

FIG. 3. Nuclear magnetic relaxation time $T_{1}$ of liquid ${ }^{3} \mathrm{He}$ containing $0.5 \%{ }^{4} \mathrm{He}$ as a function of the magnetic field for four different temperatures. The solid lines are fits to the data with Eq. (4).

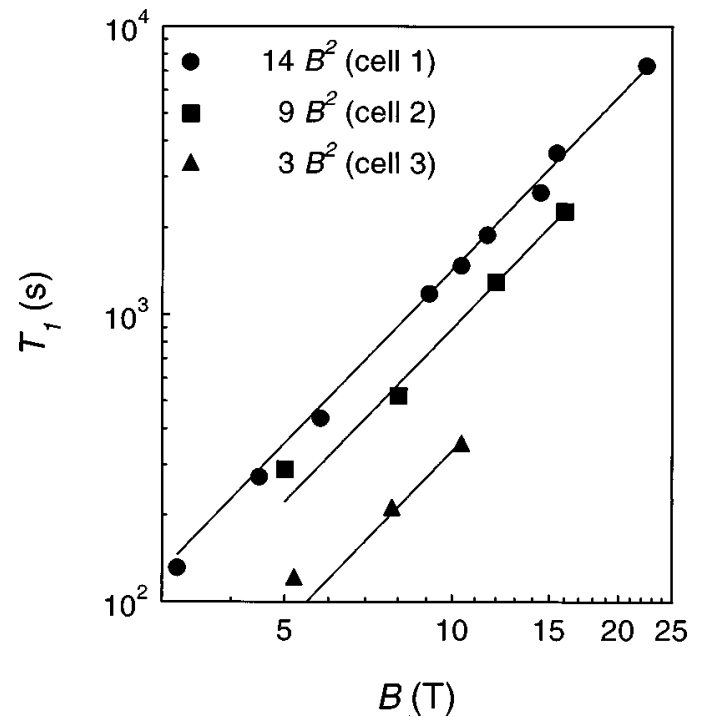

FIG. 4. Nuclear magnetic relaxation time $T_{1}$ of saturated ${ }^{3} \mathrm{He}-{ }^{4} \mathrm{He}$ mixtures at $100 \mathrm{mK}$ as a function of the magnetic field and their quadratic fits for three different cells.

magnetic-field dependence of $T_{1}$ is then due to the surface relaxation. However, since diffusion to the walls and intrinsic relaxation bound the observed $T_{1}$, respectively, at small and large values, the field dependence of the surface relaxation cannot be directly deduced from Fig. 3. On the other hand, because in these experiments the surface is covered with a saturated ${ }^{4} \mathrm{He}$ film, we can expect the surface relaxation to be identical to that for a saturated mixture at the same temperature. This brings us to the possibility of studying separately the latter phenomenon as a function of magnetic field, as, in a dilute mixture, diffusion to the walls is much faster and intrinsic relaxation is much slower than for pure ${ }^{3} \mathrm{He}$.

Figure 4 presents $T_{1}$ as a function of the magnetic field $B$ in three different cells, with different surface to volume ratios. Cell 1, which is the one also used for the experiments on the liquid ${ }^{3} \mathrm{He}$, has a volume of $3 \mathrm{~cm}^{3}$, cell 2 has a volume of $2 \mathrm{~cm}^{3}$, and cell 3 also has a volume of $3 \mathrm{~cm}^{3}$ but has not been coated with polystyrene. All cells have approximately the same surface area of $40 \mathrm{~cm}^{2}$. The striking result is the quadratic field dependence of the (surface) relaxation time for all cells studied, up to the highest fields. For the two coated cells (cell 1 and 2) $T_{1}$ is within experimental error proportional to the volume, while coating of the cell leads to an increase of $T_{1}$ by a factor of almost 5 . Both points show that the measured $T_{1}$ is dominated by surface relaxation. The observation of the $B^{2}$ dependence both in coated and in uncoated cells shows that this behavior does not depend on the details of the surface material.

In order to study the concentration dependence of the surface relaxation, we also performed experiments on ${ }^{3} \mathrm{He}-{ }^{4} \mathrm{He}$ mixtures at higher temperature so as to increase the saturation concentration. $T_{1}$ 's of ${ }^{3} \mathrm{He}-{ }^{4} \mathrm{He}$ mixtures at $300 \mathrm{mK}$ with four different ${ }^{3} \mathrm{He}$ concentrations measured in cell 2 , are plotted in Fig. 5 as a function of the magnetic field. A field-dependent relaxation time is also observed, with a saturation effect at the largest fields, which we attribute to intrinsic relaxation. Finally, we have measured in the same cell $T_{1}$ for ${ }^{3} \mathrm{He}$ concentrations ranging from 60 to $95 \%$ at a tem- 


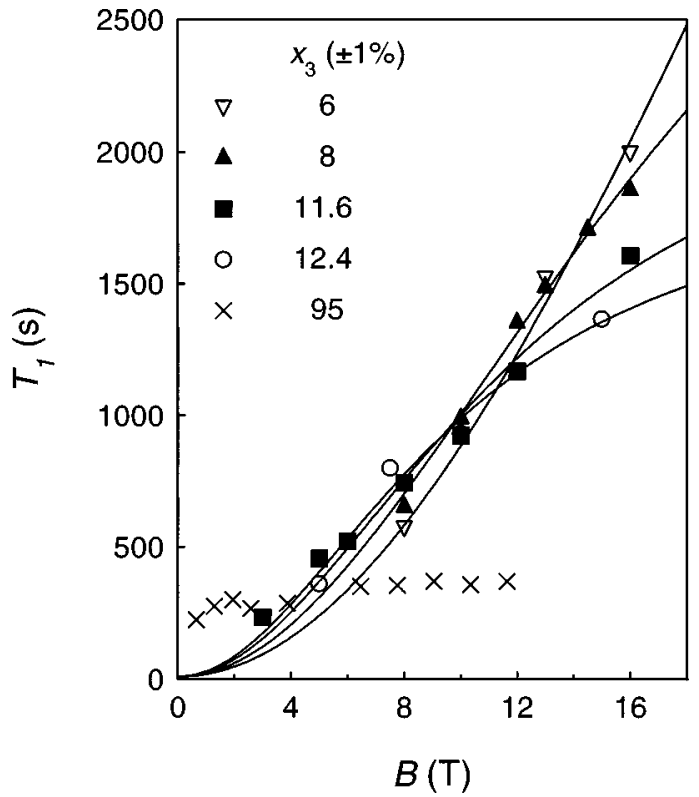

FIG. 5. Nuclear magnetic relaxation time $T_{1}$ as a function of the magnetic field of ${ }^{3} \mathrm{He}-{ }^{4} \mathrm{He}$ mixtures with four different ${ }^{3} \mathrm{He}$ concentrations $x_{3}$ at $300 \mathrm{mK}$ and $95 \%{ }^{3} \mathrm{He}$ at $1 \mathrm{~K}$. The solid lines are fits to the data with Eq. (4).

perature of $1 \mathrm{~K}$, from which only the data of the ${ }^{3} \mathrm{He}-{ }^{4} \mathrm{He}$ mixture with $95 \%{ }^{3} \mathrm{He}$ has been plotted in Fig. 5. Above $6 \mathrm{~T}$, $T_{1}$ is independent of the magnetic field, meaning that the intrinsic relaxation dominates and that $T_{\text {in }}$ can be directly determined.

\section{DISCUSSION}

One of the main conclusions that can be drawn from the results presented above, is that surface relaxation is clearly suppressed by the magnetic field. In particular, for the $6 \%$ mixture at $100 \mathrm{mK}$, where no influence of intrinsic relaxation is observed, the surface relaxation time increases with the square of the magnetic field. In the following, we first introduce a relaxation model accounting for this behavior (Secs. IV A and IV B). In this model, the field fluctuations experienced by the atoms close to the surface are characterized by a single correlation time. This correlation time sets the field scale beyond which the surface relaxation is suppressed, and its variation with temperature depends on the relaxation mechanism. We show that this model consistently accounts for the data for pure ${ }^{3} \mathrm{He}$, and that the temperature dependence of the surface relaxation rate is qualitatively consistent with the relaxation mechanism being the diffusive motion of atoms close to the surface (Sec. IV C). This model also accounts for the field dependence of the relaxation time of the mixtures at $300 \mathrm{mK}$, but, here, quantitative analysis suggests that the relaxation mechanism could differ from that at lower temperatures (Sec. IV D). Section IV E discusses in greater detail than Sec. IV C the quantitative implications on the relaxation mechanism of the results on pure ${ }^{3} \mathrm{He}$ below 200 $\mathrm{mK}$. Finally, our model allows us to extract from the field dependence of $T_{1}$ the intrinsic relaxation time $T_{\text {in }}$, of which the temperature and concentration dependences are discussed in Sec. IV F.

\section{A. Surface relaxation model}

In our relaxation model, we assume that the intrinsic relaxation is independent of the magnetic field, and that the surface relaxation takes place inside a so-called active layer and is caused by magnetic-field fluctuations near the surface. The thickness of this layer depends on the range of the field fluctuations, and is so small that the diffusion is fast enough to maintain a homogeneous magnetization within the layer.

In the case of the liquid ${ }^{3} \mathrm{He}$ with $0.5 \%{ }^{4} \mathrm{He}$, the ${ }^{4} \mathrm{He}$ forms a superfluid film on top of a solid layer of ${ }^{4} \mathrm{He}$ at the surface. $^{21,22} \mathrm{We}$ assume that the surface relaxation takes place inside this film (the thickness of the active layer is smaller than the film thickness), and that the ${ }^{3} \mathrm{He}$ concentration within the film is equal to the (temperature dependent) bulk solubility of ${ }^{3} \mathrm{He}$ in ${ }^{4} \mathrm{He}$ in zero field. In other words, we assume that the surface relaxation process in liquid ${ }^{3} \mathrm{He}$ which contains sufficient ${ }^{4} \mathrm{He}$ to cover the entire surface, is essentially the same as that in a saturated ${ }^{3} \mathrm{He}-{ }^{4} \mathrm{He}$ mixture at the same temperature. Evidence for this hypothesis will be given in Sec. IV C.

Bulk and surface relaxation are parallel processes, thus the experimental relaxation rate $T_{1}$ is given by

$$
\frac{1}{T_{1}}=\frac{1}{T_{\mathrm{s}}}+\frac{1}{T_{\mathrm{in}}},
$$

with $T_{\text {in }}$ being the intrinsic relaxation time and $T_{\mathrm{s}}$ being the surface relaxation time.

To describe the surface relaxation inside the active layer, we use the classical theory of Bloembergen, Purcell, and Pound $^{12}$ (BPP) for nuclear-spin relaxation in fluids. In this theory, the nuclear spin is subject to a randomly fluctuating perturbing magnetic field with mean-square amplitude $\left\langle\delta B^{2}\right\rangle$ and correlation time $\tau_{\mathrm{c}}$, resulting in a relaxation rate $T_{1, \mathrm{~s}}^{-1}$ :

$$
T_{1, \mathrm{~s}}^{-1}=\frac{\gamma^{2}\left\langle\delta B^{2}\right\rangle \tau_{\mathrm{c}}}{1+\left(\omega \tau_{\mathrm{c}}\right)^{2}}
$$

where $\omega$ is the Larmor frequency and $\gamma$ is the gyromagnetic ratio $(\gamma=\omega / B)$. With $\gamma \approx 2 \times 10^{8} \mathrm{rd} / \mathrm{sT}$ for ${ }^{3} \mathrm{He}, \omega \tau_{\mathrm{c}} \approx 1$ for a $\tau_{\mathrm{c}}$ of $5 \mathrm{~ns}$ at $1 \mathrm{~T}$. In principle, we also have an intrinsic relaxation process for the mixture inside the active layer, due to dipole-dipole interactions. However, as shown by the maximal $T_{1}$ observed in Figs. 4 and 5, the relaxation time associated with this process is larger than $1000 \mathrm{~s}$ below 300 $\mathrm{mK}$. This is much longer than $T_{1, \mathrm{~s}}$, as we will see, and we will henceforth ignore this contribution.

To obtain the contribution of the surface relaxation process to $T_{1}$ measured in our experiments, $T_{1, \mathrm{~s}}$ must be multiplied by $N_{\text {tot }} / N_{\mathrm{s}}$, the ratio of the total number of spins which will have to relax to the number of spins inside the active layer. This is true as long as the atoms can reach the surface by diffusion fast enough, i.e., if the diffusion time $\tau_{D}$ across the cell is much less than $T_{1, \mathrm{~s}} N_{\text {tot }} / N_{\mathrm{s}}$. If this is not the case, we deal with diffusion-limited relaxation, in which case the relaxation will be multiexponential. The rate of the slowest diffusion mode, which is the measured quantity, corresponds to an effective surface relaxation time $T_{\mathrm{s}}$, which is, within $5 \%$, given by the sum of $T_{1, \mathrm{~s}} N_{\text {tot }} / N_{\mathrm{s}}$ and $\tau_{D}$ (see the Appendix): 


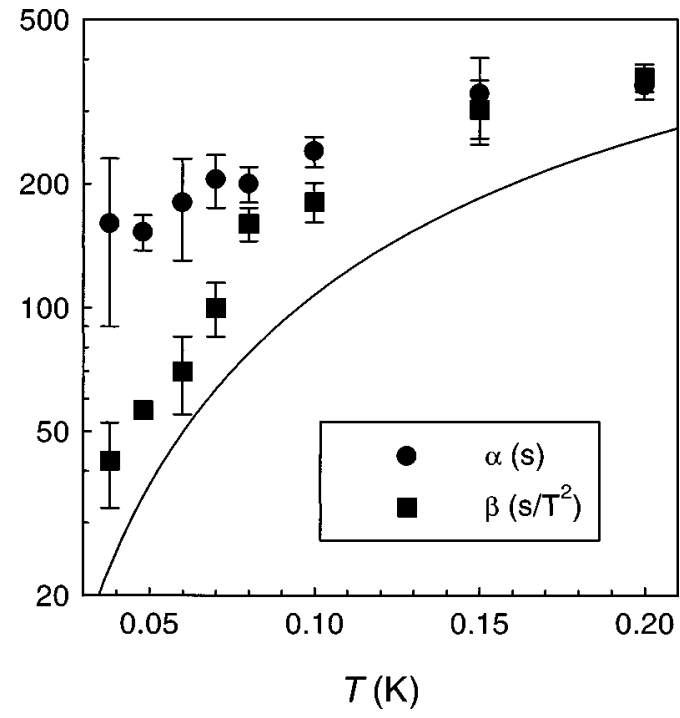

FIG. 6. The fit parameters $\alpha\left(\alpha=\tau_{D}+N_{\text {tot }} / N_{\mathrm{s}} \gamma^{2}\left\langle\delta B^{2}\right\rangle \tau_{\mathrm{c}}\right)$, and $\beta\left(\beta=N_{\text {tot }} \tau_{\mathrm{c}} / N_{\mathrm{s}}\left\langle\delta B^{2}\right\rangle\right)$ as a function of the temperature, determined with Eq. (4) from $T_{1}$ measurements of liquid ${ }^{3} \mathrm{He}$ containing $0.5 \%{ }^{4} \mathrm{He}$. The solid line is the diffusion time $\tau_{D}$ in seconds, as estimated in the text.

$$
T_{\mathrm{s}}=\tau_{D}+\frac{N_{\text {tot }}}{N_{\mathrm{s}}} \frac{1}{\gamma^{2}\left\langle\delta B^{2}\right\rangle \tau_{\mathrm{c}}}+\frac{N_{\text {tot }}}{N_{\mathrm{s}}} \frac{\tau_{\mathrm{c}}}{\left\langle\delta B^{2}\right\rangle} B^{2} .
$$

For a slab of thickness $2 L, \tau_{D}=(2 L)^{2} /\left(\pi^{2} D\right)$ where $D$ is the diffusion coefficient for liquid ${ }^{3} \mathrm{He}$, e.g., from Ref. 23. If we take $5.6 \mathrm{~mm}$ for the characteristic length $2 L$ (corresponding to the diameter of the hole in the wheel of the magnetometer), then the calculated $\tau_{D}$ increases with temperature as shown in Fig. 6. The temperature dependence of $N_{\mathrm{s}},\left\langle\delta B^{2}\right\rangle$, and $\tau_{\mathrm{c}}$ are a priori unknown. In classical liquids, where the fluctuating fields are caused by translational motion of the atoms or molecules, the correlation time $\tau_{\mathrm{c}}$ is inversely proportional to the diffusion coefficient $D .{ }^{12}$ In our case, $\tau_{\mathrm{c}}$ contains information about the dynamics of the ${ }^{3} \mathrm{He}$ atoms close to or at the surface.

Substituting the effective surface relaxation time [Eq. (3)] into Eq. (1), the measured relaxation time $T_{1}$ can be written as

$$
\frac{1}{T_{1}}=\frac{1}{\alpha+\beta B^{2}}+\frac{1}{T_{\mathrm{in}}}
$$

with

$$
\alpha=\tau_{D}+\frac{N_{\mathrm{tot}}}{N_{\mathrm{s}} \gamma^{2}\left\langle\delta B^{2}\right\rangle \tau_{\mathrm{c}}}, \quad \beta=\frac{N_{\mathrm{tot}} \tau_{\mathrm{c}}}{N_{\mathrm{s}}\left\langle\delta B^{2}\right\rangle} .
$$

In a previous paper $^{8}$ we have shown that Eq. (4) describes the magnetic-field dependence of the relaxation time measurements of saturated ${ }^{3} \mathrm{He}-{ }^{4} \mathrm{He}$ mixtures and liquid ${ }^{3} \mathrm{He}$ containing $0.5 \%{ }^{4} \mathrm{He}$.

In the following subsections we will analyze all our relaxation time measurements in the framework of Eq. (4), including those on saturated ${ }^{3} \mathrm{He}-{ }^{4} \mathrm{He}$ mixtures at $100 \mathrm{mK}$ ( $\mathrm{Sec}$. IV B), liquid ${ }^{3} \mathrm{He}$ containing $0.5 \%{ }^{4} \mathrm{He}$ (Sec. IV C), and liquid ${ }^{3} \mathrm{He}-{ }^{4} \mathrm{He}$ mixtures at $300 \mathrm{mK}$ and at $1 \mathrm{~K}$ with various
${ }^{3} \mathrm{He}$ concentrations (Sec. IV D). Furthermore, we will discuss in more detail the intrinsic relaxation time as a function of temperature and ${ }^{3} \mathrm{He}$ concentration (Sec. IV F), and the relation between diffusion and the correlation time in the surface relaxation process (Sec. IV E).

\section{B. Saturated ${ }^{3} \mathrm{He}-{ }^{4} \mathrm{He}$ mixture at $100 \mathrm{mK}$}

Let us first discuss how the results for the saturated mixtures at $100 \mathrm{mK}$ (Fig. 4) are interpreted in the framework of Eq. (4). First, our BPP-like model obviously accounts for the observed $B^{2}$ behavior. The fact that we do not see any saturation at the largest fields shows that $T_{\text {in }}$ is larger than $10^{4} \mathrm{~s}$. On the other hand, there is no saturation at the lowest fields either. From Eq. (2), this implies that $\left(\omega \tau_{\mathrm{c}}\right)^{2} \approx 1$, even at 3 $\mathrm{T}$, the lowest field studied. This means that the correlation time $\tau_{\mathrm{c}}$ is at least $2 \mathrm{~ns}$, which we will discuss below. Finally, the fact that we do not observe any saturation at the lowest fields due to the finite diffusion time $\tau_{D}$ to the walls is perfectly understood, as $\tau_{D} \approx 2 \mathrm{~s}$ for a saturated ${ }^{3} \mathrm{He}-{ }^{4} \mathrm{He}$ mixture at $100 \mathrm{mK},{ }^{24}$ much smaller than the measured $T_{1}(100$ s-2000 s).

\section{Liquid ${ }^{3} \mathrm{He}$ with $0.5 \%{ }^{4} \mathrm{He}$}

Let us now turn to the case of liquid ${ }^{3} \mathrm{He}$ with $0.5 \%{ }^{4} \mathrm{He}$. Since we assume the surface layer in this case to be identical to that of a saturated mixture, we expect that the full Eq. (4), now with nonzero $1 / T_{\text {in }}$ and $\alpha$, should fit our data. As shown by the solid lines in Fig. 3, this is indeed the case for all temperatures from 40 up to $750 \mathrm{mK}$, with $\alpha, \beta$, and $T_{\text {in }}$ as the only (temperature dependent but field independent) fit parameters. For the particular temperature $100 \mathrm{mK}$, the value found for $\beta$, which is a measure of the surface relaxation, is approximately $200 \mathrm{~s} / \mathrm{T}^{2}$, to be compared to $14 \mathrm{~s} / \mathrm{T}^{2}$ in the case of the saturated mixture in the same cell (Fig. 4). The ratio of $\beta$ 's is thus equal to ratio of $N_{\text {tot }}$, the total number of spins, in both cases. This scaling is precisely that expected if the surface contribution $T_{1, \mathrm{~s}}$ and the number of atoms in the active surface layer $N_{\mathrm{s}}$ are the same in both situations. It strongly supports our assumption that the surface relaxation takes place within the saturated film and that the active layer is essentially the same in both cases.

The quadratic field dependence of $T_{1, \mathrm{~s}}$ is in contrast with the linear field dependence generally found in ultrapure ${ }^{3} \mathrm{He}$ samples with solid ${ }^{3} \mathrm{He}$ at the surface. ${ }^{9}$ If we would fit our data using such a linear behavior, we would need a negative (unphysical) value for $\alpha$. This implies at least that such a behavior could not extend below $\sim 1 \mathrm{~T}$, unlike in the very pure system. This illustrates the critical role of the ${ }^{3} \mathrm{He}$ solid layer in determining the linear field dependence of $T_{1, \mathrm{~s}}$ in pure ${ }^{3} \mathrm{He}$ experiments. ${ }^{9}$ On the other hand, the conclusion that, for larger fields, $T_{1, \mathrm{~s}}$ behaves as $B^{2}$ rather than $B$ could not be drawn from Fig. 3 only. The experiment on the saturated mixture (Fig. 4) is essential in this respect. Due to these results, we can safely extrapolate the intrinsic relaxation time $T_{\text {in }}$ from the measured $T_{1}$, even though $T_{1}$ does not saturate in our field range at the lowest temperatures.

In order to obtain more information about the surface relaxation process, we will discuss in the rest of this subsection the temperature dependence of the two fit parameters de- 


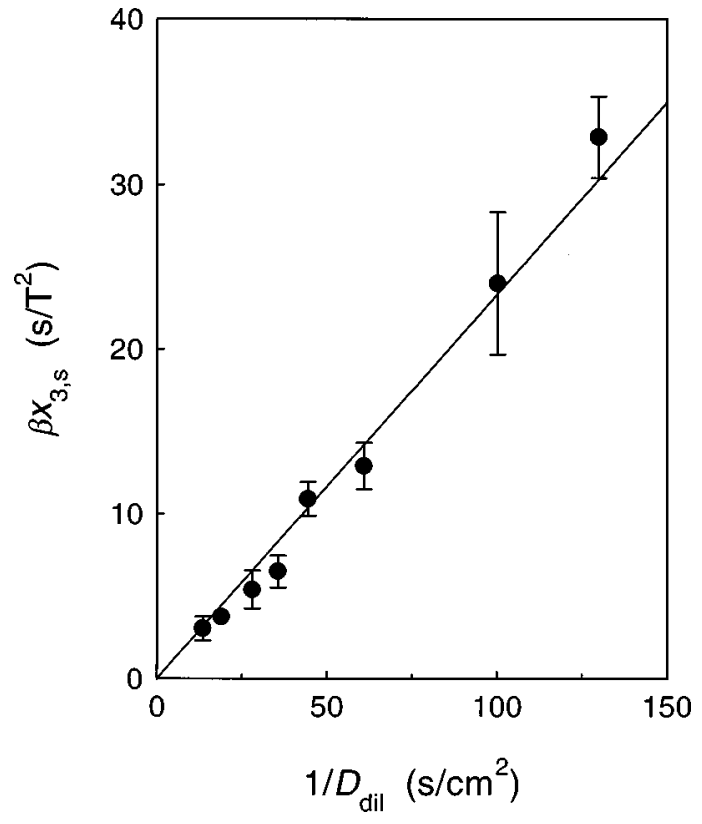

FIG. 7. $\beta x_{3, \mathrm{~s}}$ as a function of the reciprocal diffusion coefficient $D_{\text {dil }}^{-1}$ of a saturated ${ }^{3} \mathrm{He}-{ }^{4} \mathrm{He}$ mixture. $D_{\text {dil }}$ was taken from Ref. 24 for a ${ }^{3} \mathrm{He}-{ }^{4} \mathrm{He}$ mixture with $5 \%{ }^{3} \mathrm{He}$, and has been corrected for the ${ }^{3} \mathrm{He}$ concentration. The temperature ranges from $40 \mathrm{mK}$ where $D_{\text {dil }}^{-1} \approx 20 \mathrm{~s} / \mathrm{cm}^{2}$ to $200 \mathrm{mK}$ where $D_{\text {dil }}^{-1} \approx 150 \mathrm{~s} / \mathrm{cm}^{2}$.

scribing $T_{\mathrm{s}}$, which are plotted as a function of the temperature in Fig. 6. In our analysis, we will focus on $\tau_{\mathrm{c}}$, as it is this quantity which is determined by the spin dynamics. We will also restrict ourselves to $T<250 \mathrm{mK}$, since for larger temperatures, all the liquid ${ }^{4} \mathrm{He}$ is likely to be dissolved into the ${ }^{3} \mathrm{He}$, so that our picture of a saturated layer does not apply anymore. Figure 6 shows an increase of $\beta$ ( $\beta$ $\left.=N_{\text {tot }} \tau_{\mathrm{c}} / N_{\mathrm{s}}\left\langle\delta B^{2}\right\rangle\right)$ with temperature. If we assume that $\left\langle\delta B^{2}\right\rangle$ does not change at these low temperatures and high magnetic fields, the increase of $\beta$ must be attributed to $\tau_{\mathrm{c}}$, as $N_{\text {tot }}$ remains constant while $N_{\mathrm{s}}$ only slightly increases with temperature because of the increasing maximum solubility $x_{3, \mathrm{~s}}$ of ${ }^{3} \mathrm{He}$ in the ${ }^{4} \mathrm{He}$ film $\left[x_{3, \mathrm{~s}}\right.$ increases from $6.6 \%$ at 40 $\mathrm{mK}$ to $7 \%$ at $100 \mathrm{mK}$ and $9 \%$ at $200 \mathrm{mK}$ (Ref. 25)].

This increase of the correlation time $\tau_{\mathrm{c}}$ is expected if the motion responsible for relaxation is the diffusion of atoms close to the surface. In order to test more quantitatively this idea, we plotted in Fig. $7 \beta(T) x_{3, \mathrm{~s}}(T)$ as a function of $D_{\text {dil }}^{-1}(T)$, where $D_{\text {dil }}(T)$ is the diffusion constant of a saturated dilute mixture. The $x_{3, \mathrm{~s}}(T)$ factor cancels out the $N_{\mathrm{s}}$ dependence of $\beta$, so that $\beta(T) x_{3, \mathrm{~s}}(T)$ is proportional to $\tau_{\mathrm{c}}$. We obtained $D_{\text {dil }}(T)$ from the measured values ${ }^{24}$ for a ${ }^{3} \mathrm{He}-{ }^{4} \mathrm{He}$ mixture with a concentration of $5 \%{ }^{3} \mathrm{He}$ by correcting for the ${ }^{3} \mathrm{He}$ concentration $\left(D \sim x_{3}^{2 / 3}\right.$ in the degenerate regime $\left.^{24}\right)$. The proportionality of $\beta x_{3, \mathrm{~s}}$ to $D_{\text {dil }}^{-1}$ means that they have the same temperature dependence. This is indeed consistent with the idea that $\tau_{\mathrm{c}} \sim D_{\text {dil }}^{-1}$, i.e., that relaxation occurs through the diffusive motion of ${ }^{3} \mathrm{He}$ atoms inside the saturated layer.

This behavior provides a natural explanation for the previously unexplained observations ${ }^{2}$ that $T_{\mathrm{s}}$ is inversely proportional to the diffusion constant $D$ of pure ${ }^{3} \mathrm{He}$ in experiments where $T_{\mathrm{s}}$ is clearly larger than $\tau_{D}$, the diffusion time across the sample. Indeed, in these conditions, $T_{\mathrm{s}} \sim \beta$ (at large enough field) $\sim D^{-1}$, since the diffusion constant for pure and dilute ${ }^{3} \mathrm{He}$ are approximately proportional to one another below $200 \mathrm{mK}$.

An absolute estimate of $\tau_{\mathrm{c}}$ can be obtained from the combined values of $\alpha$ and $\beta$. According to Eq. (5)

$$
\alpha=\tau_{D}(T)+\frac{\beta}{\left(\gamma \tau_{\mathrm{c}}\right)^{2}}=\tau_{D}(T)+\left(\frac{N_{\text {tot }}}{N_{\mathrm{s}} \gamma\left\langle\delta B^{2}\right\rangle}\right)^{2} \frac{1}{\beta},
$$

with $\tau_{D}=(2 L)^{2} / \pi^{2} D(T)$, where $2 L$ is of the order of cell size. If we again assume that $N_{\text {tot }} / N_{\mathrm{s}}\left\langle\delta B^{2}\right\rangle$ is independent of temperature, $\alpha$ can reasonably be described as a linear function of $1 / \beta$, with $L$ and $N_{\text {tot }} /\left(N_{\mathrm{s}} \gamma\left\langle\delta B^{2}\right\rangle\right)^{2}$ as free parameters. This gives $2 L=6 \mathrm{~mm}$, a reasonable value since it is in between the diameter of the hole in the wheel and that of the cylindrical cell. With this value of $L, \alpha$ is dominated by the contribution of $\tau_{D}$. This explains why $\alpha$ does not increase (as $1 / \beta$ does) at low temperature. Once $L$ is determined, we get $\tau_{\mathrm{c}}$ from $\left(\gamma \tau_{\mathrm{c}}\right)^{2}=\beta /\left(\alpha-\tau_{D}\right) . \tau_{\mathrm{c}}$ is found to increase from $4 \mathrm{~ns}$ up to $12 \mathrm{~ns}$ from $T=40$ to $100 \mathrm{mK}$. The relative increase is less than for $D_{\text {dil }}^{-1}$ in the same temperature range, but this could be due to the poor precision on $\alpha-\tau_{D}$, which makes it difficult to determine $\tau_{\mathrm{c}}$ to better than $2 \mathrm{~ns}$.

Even if we do not make the previous assumption that $\alpha$ and $\beta$ only depend on temperature through $\tau_{\mathrm{c}}$, we can still give a lower bound for $\tau_{\mathrm{c}}$ since $2 L \approx 3 \mathrm{~mm}$ is certainly a lower limit for evaluating $\tau_{D}$. At $100 \mathrm{mK}$, this gives $\tau_{\mathrm{c}}>5$ ns. This estimate is consistent with that inferred from the study of the saturated mixture at $100 \mathrm{mK}$. We defer the discussion of this order of magnitude to Sec. IV E.

\section{D. ${ }^{3} \mathrm{He}-{ }^{4} \mathrm{He}$ mixtures at $300 \mathrm{mK}$ and $1 \mathrm{~K}$}

We saw in the previous subsection that our relaxation model, with a correlation time proportional to $1 / D_{\text {dil }}$, accounted for measurements of $T_{1}$ in ${ }^{3} \mathrm{He}$ with $0.5 \%{ }^{4} \mathrm{He}$. What should we expect, within the same model, for our data on mixtures at $300 \mathrm{mK}$ ? If the temperature dependence of $\alpha$ and $\beta$ is only through $\tau_{\mathrm{c}}$ (and $\tau_{D}$ ) in Eq. (5), their values at $300 \mathrm{mK}$ for the mixtures can be deduced from the measurements of the $6 \%$ mixture at $100 \mathrm{mK}$ in the same cell (cell 2) by scaling $\beta$ as $1 / \tau_{\mathrm{c}}$ and using Eq. (6) for $\alpha$ (in our model of an active layer of fixed thickness, the ratio $N_{\text {tot }} / N_{\mathrm{s}}$ does not depend on the dilute mixture concentration). As $D_{\mathrm{dil}} \approx 3.5$ $\times 10^{-3} \mathrm{~cm}^{2} / \mathrm{s}$ at $300 \mathrm{mK}$ and $D_{\text {dil }} \approx 15 \times 10^{-3} \mathrm{~cm}^{2} / \mathrm{s}$ at 100 $\mathrm{mK}$ for a $5 \%$ mixture, ${ }^{24}$ we expect $\tau_{\mathrm{c}}$ to be approximately four times larger for the $6 \%$ mixture at $300 \mathrm{mK}$ than at 100 $\mathrm{mK}$, i.e., $\tau_{\mathrm{c}}>8 \mathrm{~ns}$ (see Sec. IV B). For the $11.6 \%$ mixture, $\tau_{\mathrm{c}}$ should be increased by a further factor of 2 , as $D_{\mathrm{dil}} \sim 1 / x_{3}$ in this temperature range, ${ }^{24}$ i.e., $\tau_{\mathrm{c}}>16 \mathrm{~ns}$. As $\beta \approx 9 \mathrm{~s} / \mathrm{T}^{2}$ at $100 \mathrm{mK}$ in cell $2, \beta$ should correspondingly range from 40 to $80 \mathrm{~s} / \mathrm{T}^{2}$ at $300 \mathrm{mK}$ for mixtures of concentration $6-12 \%$. $\beta /\left(\gamma \tau_{\mathrm{c}}\right)^{2}$ should then be smaller than $15 \mathrm{~s}$. For the above $D_{\text {dil }}$ values, $\tau_{D}$ is also less than $10 \mathrm{~s}$ (using $2 L=6 \mathrm{~mm}$ as an estimate of the distance between relaxing walls), so that $\alpha$ should be less than $25 \mathrm{~s}$.

In our $T_{1}$ range $(500-2000 \mathrm{~s})$, we finally expect $1 / T_{1}=1 / T_{\text {int }}+1 /\left(\beta B^{2}\right)$. Accordingly, we plot in Fig. $81 / T_{1}$ as a function of $1 / B^{2}$. These coordinates, unlike those of Fig. 4 , allow for the non-negligible bulk relaxation rate at these temperature and concentrations. The data of the mixtures 


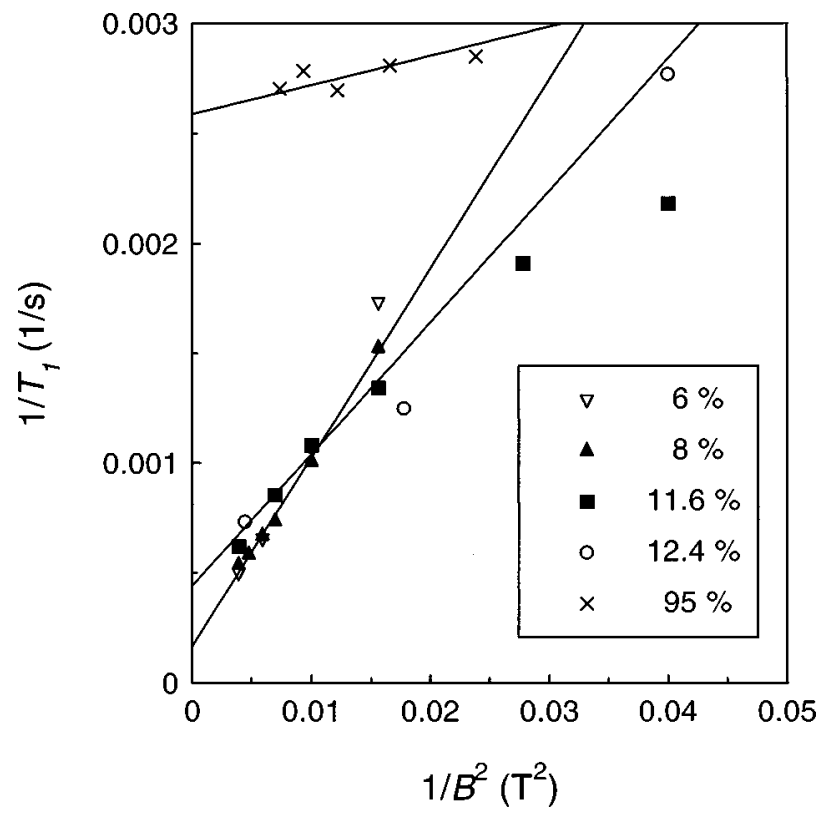

FIG. 8. Relaxation rate $1 / T_{1}$ as a function of the square of the reciprocal field $1 / B^{2}$ of ${ }^{3} \mathrm{He}-{ }^{4} \mathrm{He}$ mixtures with four different ${ }^{3} \mathrm{He}$ concentrations $x_{3}$ at $300 \mathrm{mK}$ and $95 \%{ }^{3} \mathrm{He}$ at $1 \mathrm{~K}$, as denoted in the legend. Lines are fits as described in the text.

with $(7 \pm 1) \%{ }^{3} \mathrm{He}$ and those with $(12 \pm 1) \%{ }^{3} \mathrm{He}$ have been fitted as a linear function of $1 / B^{2}$ for $B$ larger than $8 \mathrm{~T}$, and the results are shown as straight lines in Fig. 8. The values for $\beta$ resulting from the slope of these straight lines range from $12 \pm 1 \mathrm{~s} / \mathrm{T}^{2}$ to $17 \pm 1 \mathrm{~s} / \mathrm{T}^{2}$, that is, do not show the fourfold increase predicted from the expected change in $\tau_{\mathrm{c}}$. The $11.6 \%$ data, which extend to the lowest fields, clearly deviate from a straight line. Accordingly, these data can only be fitted to Eq. (4) using a larger value of $\alpha(\alpha \approx 100$ s) than expected.

The discrepancy between the observed and predicted values of $\alpha$ and $\beta$ cannot result from an extra temperature dependence, e.g., through $\left\langle\delta B^{2}\right\rangle$, of $\alpha$ and $\beta$ from 100 to 300 $\mathrm{mK}$, since $\alpha$ is too large whereas $\beta$ is too small with respect to the predicted values. As an effect, $\tau_{\mathrm{c}}$ estimated from $\left(\gamma \tau_{\mathrm{c}}\right)^{2}=\beta /\left(\alpha-\tau_{D}\right)$ is of order of $2 \mathrm{~ns}$ (independently of any hypothesis on the constancy of $\left\langle\delta B^{2}\right\rangle$ with temperature), ten times smaller than expected from the assumed scaling $\tau_{\mathrm{c}}$ $\sim 1 / D_{\text {dil }}$. This may mean that another relaxing mechanism becomes more efficient than the diffusive motion of atoms at $300 \mathrm{mK}$. In the future, it would be desirable to study continuously the evolution of $T_{1}$ for a $6 \%$ mixture from low temperatures up to $300 \mathrm{mK}$.

Despite this problem, the data at large fields show a reasonable linear behavior of $1 / T_{1}$ as a function of $1 / B^{2}$. This allows to extract $T_{\text {in }}$ for the mixtures at $300 \mathrm{mK}$ as a function of ${ }^{3} \mathrm{He}$ concentration. The values found are shown in Fig. 10 and discussed in Sec. IV F.

\section{E. $\tau_{\mathrm{c}}$ and the mechanism of surface relaxation}

In the following, we focus on our results below $200 \mathrm{mK}$ for ${ }^{3} \mathrm{He}$ with $0.5 \%{ }^{4} \mathrm{He}$, for which a scaling of $\beta$ with $1 / D_{\text {dil }}$ is observed. As discussed in Sec. IV C, this scaling is consistent with the hypothesis that relaxation occurs through dif- fusive motion of the ${ }^{3} \mathrm{He}$ atoms within the ${ }^{4} \mathrm{He}$ film close to the surface. Here, we analyze the significance of our quantitative results on the correlation time $\tau_{\mathrm{c}}$, in the framework of both the above hypothesis, and of alternate explanations for the observed scaling. A comparison to surface relaxation on other substrates is also presented.

In the case where ${ }^{3} \mathrm{He}$ spins relax due to diffusive motion in the inhomogeneous magnetic field of the surface, $\tau_{\mathrm{c}}$ $=l^{2} / \pi^{2} D_{\text {dil }}$, with $l$ being the correlation length of the inhomogeneous field and $D_{\text {dil }}$ being the diffusion coefficient of the ${ }^{3} \mathrm{He}$ in the ${ }^{3} \mathrm{He}-{ }^{4} \mathrm{He}$ mixture film near the surface. In Sec. IV C, we showed that $\tau_{\mathrm{c}}>5 \mathrm{~ns}$ at $100 \mathrm{mK}$, independently of any hypothesis on the mechanism of surface relaxation. If we take for $D_{\text {dil }}$ the bulk value of a $5 \%{ }^{3} \mathrm{He}-{ }^{4} \mathrm{He}$ mixture,$^{24}$ this corresponds to a correlation length larger than $0.25 \mu \mathrm{m}$, which is about four times the reported ${ }^{4} \mathrm{He}$ film thickness. ${ }^{21}$ As the results of Secs. IV B and IV C are consistent with the idea that the thickness of the active layer is itself smaller than that of the film (scaling of $\beta$ ), we end up with a correlation length larger than this thickness, i.e., larger than the range of field fluctuations, perpendicular to the surface. It is not clear to us whether this is possible or not. In any case, this value of $l$ is not large enough, taking into account the possible uncertainty in the film thickness or the possible change of the diffusion constant close to the surface, to rule out spin diffusion as the relaxing mechanism.

An alternative hypothesis to explain the observed long correlation time might be that a ${ }^{3} \mathrm{He}$ atom sticks to the surface and relaxes due to the perturbing field of a paramagnetic impurity at the surface of the cell, in a similar way to that observed for ${ }^{3} \mathrm{He}$ gas, in hydrogen coated cells in the lowfield limit. ${ }^{26} N_{\mathrm{s}}$ would then correspond to the number of ${ }^{3} \mathrm{He}$ atoms at the surface, and $\tau_{\mathrm{c}}$ to the sticking time.

Measuring the temperature dependence of $\tau_{\mathrm{c}}$ should allow us to discriminate between the two mechanisms. $\tau_{\mathrm{c}}$ should increase with temperature in the first mechanism (relaxation due to diffusion), whereas it should stay constant, or even decrease with increasing temperature in the second mechanism (relaxation due to sticking). As discussed in Sec. IV C, the temperature variation of $\tau_{\mathrm{c}}$ cannot be unambiguously determined in the case of almost pure ${ }^{3} \mathrm{He}$, due to the large uncertainty of $\tau_{D}$ in Eq. (6), so that both mechanisms are in fact possible. In the second mechanism, the experimentally measured increase of $\beta$ with temperature should then be attributed either to a decrease of $N_{\mathrm{s}}$, or to a decrease of the field fluctuations, with increasing temperature. Experiments studying the temperature dependence of $T_{\mathrm{s}}$ for a saturated mixture could possibly discriminate between the two mechanisms, by allowing us to measure directly the temperature dependence of $\tau_{\mathrm{c}}$. Indeed, with respect to the case of almost pure ${ }^{3} \mathrm{He}, \tau_{D}$ would be reduced by a factor of 50 , whereas $\alpha-\tau_{D}$ would be only reduced by the ratio of $N_{\text {tot }}$, i.e., approximately 14 , thereby increasing the relative precision on $\alpha-\tau_{D}$, and hence $\tau_{\mathrm{c}}$.

In this context of the relaxation mechanism, we remark that Eq. (2) is a classical one, where no allowance is made for phase-space restrictions imposed by the Pauli principle, as is the case for bulk liquid ${ }^{3} \mathrm{He}$. Such a restriction could give an extra temperature dependence to $T_{1, \mathrm{~s}}$, which could make $\beta$ (respectively, $\alpha$ ) to vary differently from $\tau_{\mathrm{c}}$ (respec- 


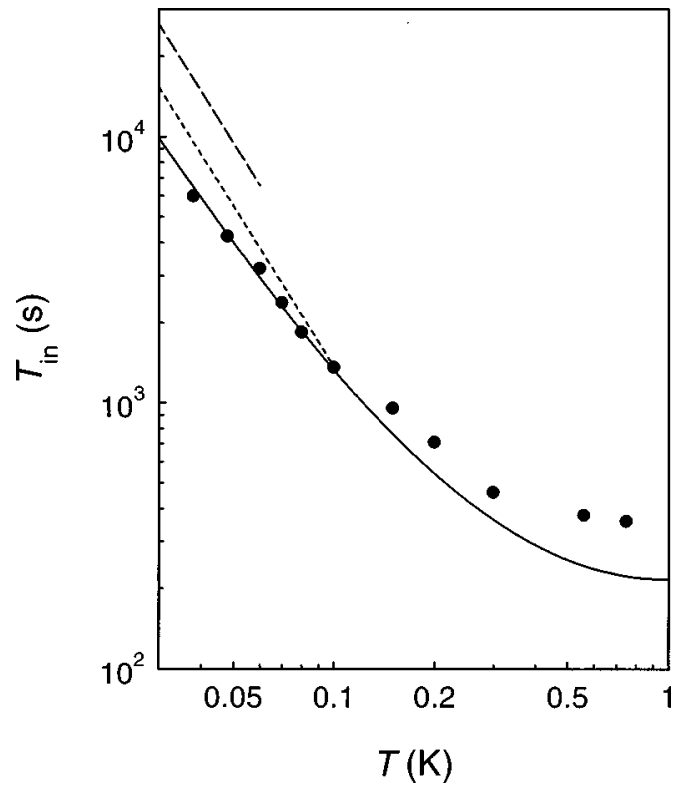

FIG. 9. Intrinsic relaxation time $T_{\text {in }}$ of liquid ${ }^{3} \mathrm{He}$ as a function of temperature determined with Eq. (4). The lines represent the theory of Vollhardt and Wölfle (solid line, Ref. 5), Bedell and Melzer (lower dashed line, Ref. 6), and Havens-Sacco and Widom (upper dashed line, Ref. 7).

tively, $\left.1 / \tau_{\mathrm{c}}\right)$. To our knowledge, this effect has not been considered previously. However, even if it does exist, it could not explain alone the temperature dependence of $\beta$, as it should probably lead to an increase of relaxation as the temperature increases.

Let us finally discuss the absolute strength of surface relaxation, independently of any hypothesis on its mechanism. The surface relaxation time is $T_{1, \mathrm{~s}}=\left(T_{\mathrm{s}}-\tau_{D}\right) N_{\mathrm{s}} / N_{\text {tot }}=(\alpha$ $\left.+\beta B^{2}-\tau_{D}\right) N_{\mathrm{s}} / N_{\mathrm{tot}}$. Depending on the thickness of the active layer, which may range from one atomic layer up to $l$, $N_{\mathrm{s}} / N_{\text {tot }}$ ranges from $\sim 2 \times 10^{-8}$ to $\sim 2 \times 10^{-5}$ for cell 1 , taking $6 \%$ as the concentration inside the saturated film. Thus, a typical $T_{\mathrm{s}}$ of $1000 \mathrm{~s}$ in $3 \mathrm{~T}\left(\beta=100 \mathrm{~s} / \mathrm{T}^{2}\right)$ corresponds to $T_{1, \mathrm{~s}}$ from $20 \mu \mathrm{s}$ up to $20 \mathrm{~ms}$. These numbers are short with respect to those reported for pure ${ }^{3} \mathrm{He}$ on surfaces of high specific area [typically $1 \mathrm{~s}$ in $3 \mathrm{~T}$ (Refs. 9 and 27)] where the relaxation is believed to occur through exchange between the first solid layer and the liquid, and where addition of ${ }^{4} \mathrm{He}$ considerably reduces the relaxation. ${ }^{9}$ This suggests that the field fluctuations associated with a plastic material could be much larger than for these other substrates. From $\beta, \tau_{\mathrm{c}}$, and $N_{\mathrm{s}} / N_{\text {tot }}$, we can estimate $\left\langle\delta B^{2}\right\rangle$ from Eq. (5). Using $\beta=100 \mathrm{~s} / \mathrm{T}^{2}, \tau_{\mathrm{c}}>5 \mathrm{~ns}$ at $100 \mathrm{mK}$, we find that $\left(\left\langle\delta B^{2}\right\rangle\right)^{1 / 2}$ ranges from $15 \times 10^{-4} \mathrm{~T}$ (for a $0.25 \mu \mathrm{m}$ thick relaxing layer) up to $0.05 \mathrm{~T}$ (for an atomic size relaxing layer). Such large values point to a high concentration of (electronic) paramagnetic impurities in the plastic walls of our cells.

\section{F. Intrinsic relaxation}

Figure 9 shows the intrinsic relaxation time $T_{\text {in }}$ of pure ${ }^{3} \mathrm{He}$, as a function of temperature, as determined with Eq. (4) from $T_{1}$ measurements of liquid ${ }^{3} \mathrm{He}$ with $0.5 \%{ }^{4} \mathrm{He}$. The experimental data have been obtained from $T_{1}$ measurements as a function of magnetic field, with the only assumption that $T_{\mathrm{s}}=\alpha+\beta B^{2}$, which, as we have shown above, describes the whole set of data of the ${ }^{3} \mathrm{He}$ excellently. Moreover, above $100 \mathrm{mK}$, the applied field was high enough to reach $T_{\text {in }}$ within experimental accuracy of about $10 \%$.

The experimentally determined $T_{\text {in }}$ has been compared with theoretical predictions of Vollhardt and Wölfle, ${ }^{5}$ Bedell and Melzer, ${ }^{6}$ and Havens-Sacco and Widom. ${ }^{7}$ As the measurements are performed at saturated vapor pressure, which is about 0 bar at these low temperatures, the theories of $\mathrm{Be}$ dell and Melzer, and Havens-Sacco and Widom, have been plotted in Fig. 9 for a pressure of 0 bar, for which we have used the Fermi-liquid parameters of Ref. 28. The theory of Havens-Sacco and Widom is in clear disagreement with the experimental data. Although the theoretical prediction of Bedell and Melzer is quantitatively in good agreement with the experiment around $100 \mathrm{mK}$, the predicted pure $T^{-2}$ dependence is not observed at these temperatures.

Excellent agreement between the experimental data and the theory of Vollhardt and Wölfle is observed in the Fermiliquid regime (below $100 \mathrm{mK}$ ). Although the qualitative temperature dependence describes quite well the transition from the Fermi-liquid regime to the Boltzmann regime above $1 \mathrm{~K}$, in the high-temperature plateau, the experimentally determined $T_{\text {in }}$ is 1.5 times longer than the theoretical value. This is in agreement with the $T_{1}$ measurements of Romer, ${ }^{29}$ which were, although not corrected for wall relaxation, also higher than the theoretical prediction. In the theory of Vollhardt and Wölfle, the total intrinsic relaxation rate is the sum of the relaxation rates in the collisionless $\left(T_{\mathrm{RPA}}^{-1}\right)$ and in the hydrodynamic regime $\left(T_{\text {dif }}^{-1}\right)$. Its calculation involves a momentum integral which contains a cutoff wave vector $q_{0}$ that separates the collisionless from the diffusive regime, and determines the high-temperature value. Because the cutoff wave vector $q_{0}$, which is proportional to the inverse mean free path, is determined only up to a factor of 2, at best, the observed difference between experiment and theory can be accounted for by the value of $q_{0}$ in the theory, and agreement between theory and experiment could be obtained by adjusting the value of $q_{0} \cdot{ }^{30}$

In Fig. 10 we have plotted the $T_{\text {in }}^{-1}$ of ${ }^{3} \mathrm{He}-{ }^{4} \mathrm{He}$ mixtures at $1 \mathrm{~K}$, as a function of the ${ }^{3} \mathrm{He}$ molar concentration $x_{3}$. For these high densities and temperature, the magnetic field was high enough to reach $T_{\text {in }}$ within the experimental accuracy of about $7 \%$, while for $T_{\text {in }}$ for the ${ }^{3} \mathrm{He}$ density of $100 \%$ we have taken the extrapolated value from Fig. 9. The straight line perfectly fits the data, showing clearly the proportionality of $T_{\text {in }}^{-1}$ to the ${ }^{3} \mathrm{He}$ concentration, and the expected behavior that $T_{1}$ goes to infinity as $x_{3}$ goes to zero.

These measurements can be compared to the prediction of Lowe et al., ${ }^{4}$ who have calculated $T_{\text {in }}$ of ${ }^{3} \mathrm{He}$ in ${ }^{3} \mathrm{He}-{ }^{4} \mathrm{He}$ mixtures for temperatures much greater than the Fermi temperature: $T_{\text {in }}^{-1}=x_{3} /\left(11 T^{1 / 2}\right)$ where $T_{\text {in }}$ has the units of hours and $x_{3}$ is the concentration of ${ }^{3} \mathrm{He}$ in percent. The theoretical $T_{\text {in }}^{-1}$, which has been plotted for $T=1 \mathrm{~K}$ in Fig. 10, predicts the correct ${ }^{3} \mathrm{He}$ density dependence and deviates only by $10 \%$ from the experimental results.

We also have plotted $T_{\text {in }}^{-1}$ determined with Eq. (4) from $T_{1}$ of the ${ }^{3} \mathrm{He}-{ }^{4} \mathrm{He}$ mixtures at $300 \mathrm{mK}$ in Fig. 10. These data also show an increase of $T_{\text {in }}^{-1}$ with the ${ }^{3} \mathrm{He}$ density, but 


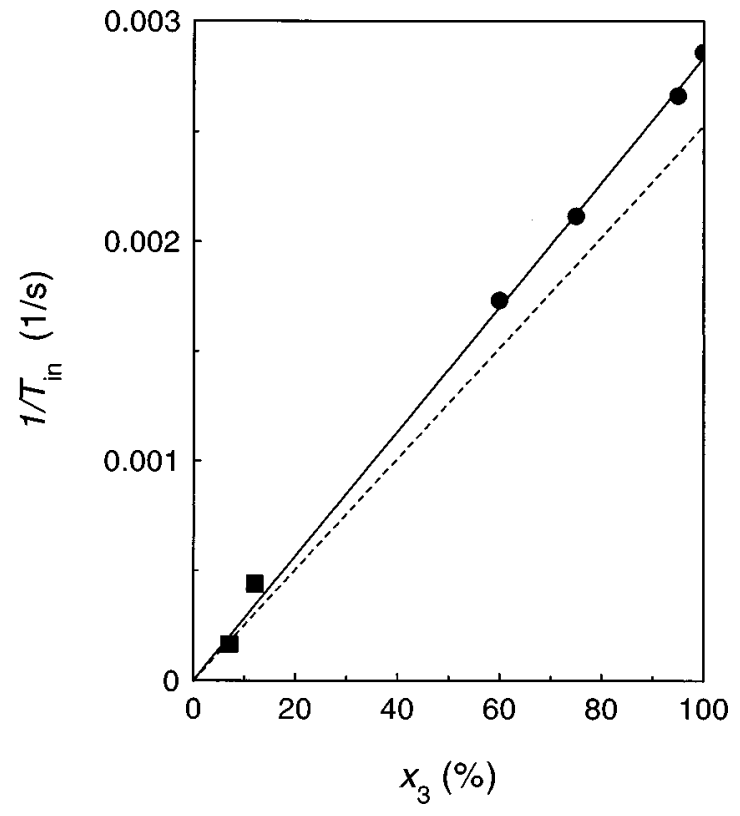

FIG. 10. Intrinsic relaxation rate $1 / T_{\text {in }}$ of ${ }^{3} \mathrm{He}-{ }^{4} \mathrm{He}$ mixtures at 1 $\mathrm{K}$ (dots) and $300 \mathrm{mK}$ (squares) as a function of the ${ }^{3} \mathrm{He}$ concentration $x_{3}$. The solid line is a fit to the $1 \mathrm{~K}$ data, the dashed line represents the theory of Lowe et al., Ref. 4.

cannot be compared with the theory of Lowe et al., since the temperature is too low $\left(T_{F} \approx 500 \mathrm{mK}\right.$ for a $10 \%{ }^{3} \mathrm{He}^{4} \mathrm{He}$ mixture ${ }^{25}$ ). The observed increase of $T_{\text {in }}^{-1}$ with the ${ }^{3} \mathrm{He}$ concentration in ${ }^{4} \mathrm{He}$ in this case is qualitatively similar to that of ${ }^{3} \mathrm{He}$ gas, for which Mullin, Laloë, and Richards ${ }^{31}$ argue that at low temperatures, but still in the Boltzmann regime, the thermal wavelength $\lambda\left[\lambda=\hbar /\left(m k_{B} T\right)^{1 / 2}\right.$, with $m$ the effective mass] becomes larger than the distance of closest approach $a$, resulting in $T_{\text {in }}^{-1} \sim n T^{1 / 2}$ where $n$ is the ${ }^{3} \mathrm{He}$ gas density. However, due to the proximity of the degenerate regime, no quantitative agreement will be considered here.

An interesting point which should be mentioned here is the prediction of a minimum for $T_{\text {in }}$ of ${ }^{3} \mathrm{He}$ gas as a function of the temperature. ${ }^{32}$ The change from $T_{\mathrm{in}} \sim T^{1 / 2}$ predicted and observed by Chapman and Richards $\mathrm{s}^{33,34}$ at high temperatures, to $T_{\text {in }} \sim T^{-1 / 2}$ at low temperatures, results in a minimum of $T_{\text {in }}$ at intermediate temperature for which $\lambda \approx a$ at about $0.8 \mathrm{~K} \cdot{ }^{32}$ At these temperatures the ${ }^{3} \mathrm{He}$ gas density becomes so low $\left(\approx 10^{25} \mathrm{~m}^{-3}\right)$ that $T_{\text {in }}$ is very long. Due to condensation and wall sticking, wall relaxation becomes the dominant relaxation process as the temperature is lowered. Therefore the minimum of $T_{\text {in }}$ could not be observed in gaseous ${ }^{3} \mathrm{He} .{ }^{35}$

In liquid ${ }^{3} \mathrm{He}$, the crossover would occur in the same temperature range as that from the classical to the degenerate regime (since $a$ is of the order of the interatomic distance $n^{-1 / 3}$ ), so that the minimum indeed observed around $1 \mathrm{~K}$ is then due to the crossover from the high-temperature Boltzmann behavior to the degenerate regime, for which one expects $T_{\text {in }} \sim T^{-2}$. On the other hand, observation of the predicted minimum seems possible for dilute solutions of ${ }^{3} \mathrm{He}$ in ${ }^{4} \mathrm{He},{ }^{20}$ using our technique to determine $T_{\text {in }}$. For a ${ }^{3} \mathrm{He}-{ }^{4} \mathrm{He}$ mixture with $10 \%{ }^{3} \mathrm{He}$, the interatomic distance $n^{-1 / 3} \approx 8 \AA$. At the predicted minimum temperature of $1 \mathrm{~K}$, $\lambda \approx a \approx 2.5 \AA$, so that $n \lambda^{3} \approx 0.04$ for such a mixture. Hence, for ${ }^{3} \mathrm{He}$ concentrations lower than $10 \%$, we indeed would have $\lambda<n^{-1 / 3}$, while the system goes from $\lambda<a$ to $\lambda>a$ at $T \approx 1 \mathrm{~K}$, thereby avoiding degeneracy.

\section{CONCLUSIONS}

We have presented a comprehensive experimental study on the nuclear magnetic relaxation time $T_{1}$ of almost pure liquid ${ }^{3} \mathrm{He}$ and ${ }^{3} \mathrm{He}-{ }^{4} \mathrm{He}$ mixtures as a function of ${ }^{3} \mathrm{He}$ concentration, temperature, and magnetic field. All results can be explained by a single model, in which the surface and intrinsic contributions to $T_{1}$ can be separated due to their different magnetic-field dependences. We have shown that the surface relaxation time of liquid ${ }^{3} \mathrm{He}$ containing ${ }^{4} \mathrm{He}$, unlike when there is a solid layer of ${ }^{3} \mathrm{He}$ at the surface, is proportional to $1+\left(\gamma B \tau_{\mathrm{c}}\right)^{2}$. The correlation time $\tau_{\mathrm{c}}$, determined from our experiments, is of the order of $5 \mathrm{~ns}$ at 100 $\mathrm{mK}$. The temperature dependence of this surface term agrees with the idea that the relaxation arises from the diffusive motion of the ${ }^{3} \mathrm{He}$ atoms in the ${ }^{4} \mathrm{He}$ film at the surface. The intrinsic relaxation time $T_{\text {in }}$ of ${ }^{3} \mathrm{He}$ we have experimentally determined with high precision, is in good agreement with the theoretical predictions by Vollhardt and Wölfle. ${ }^{5} \mathrm{We}$ have shown that $T_{\text {in }}$ in ${ }^{3} \mathrm{He}-{ }^{4} \mathrm{He}$ mixtures at a temperature of $1 \mathrm{~K}$, is proportional to the ${ }^{3} \mathrm{He}$ concentration, in agreement with theoretical predictions. ${ }^{4}$

\section{ACKNOWLEDGMENTS}

We would like to thank Jos Rook for technical support, and Kevin Bedell, Reijer Jochemsen, and Dieter Vollhardt for helpful discussions. This work is part of the research program of the "Stichting voor Fundamenteel Onderzoek der Materie" (F.O.M.) which is financially supported by the "Nederlandse Organisatie voor Wetenschappelijk Onderzoek" (The Netherlands). This work is supported by the European Community under H.C.M. Contract No. CHGECT930051 and under T.M.R. Contract No. FMGECT950079.

\section{APPENDIX}

In this appendix we calculate the total relaxation time $T_{1}$ due to intrinsic and surface relaxation. The atoms reach the surface by diffusion, of which the effect will be included in the effective surface relaxation time. We consider a fluid with a diffusion coefficient $D$, and an intrinsic relaxation time $T_{\mathrm{in}}$. The liquid is confined between two infinitely long parallel plates at a distance $x= \pm L$. We assume the surface relaxation to take place in an active layer of thickness $l_{\mathrm{a}}$, with a surface relaxation time $T_{1, \mathrm{~s}}$.

Inside the active layer, the change in magnetization $m$ is due to the surface relaxation and a flux of magnetization diffusing into this layer:

$$
\frac{\partial m}{\partial t}=\frac{m_{\mathrm{eq}}-m}{T_{1, \mathrm{~s}}}+D \frac{\partial^{2} m}{\partial x^{2}}
$$

At the boundary of the layer the magnetization flux should be continuous: 


$$
D \frac{\partial m^{-}}{\partial x}=D \frac{\partial m^{+}}{\partial x},
$$

where $m^{-}=m\left(L-l_{\mathrm{a}}-\delta\right)$ and $m^{+}=m\left(L-l_{\mathrm{a}}+\delta\right)$, while $\delta$ goes to zero. In our experimental conditions diffusion in the layer is fast enough to maintain a homogeneous magnetization $m$ in the layer (which means that $l_{\mathrm{a}}^{2} / D \ll T_{1, \mathrm{~s}}$ ). Integration of Eq. (A1) from $L-l_{\mathrm{a}}+\delta$ to $L$ results in

$$
-D \frac{\partial m^{-}}{\partial x}=l_{\mathrm{a}}\left(\frac{m}{T_{1, \mathrm{~s}}}+\frac{\partial m}{\partial t}\right),
$$

where we have used the continuity of flux [Eq. (A2)], and for simplicity set $m_{\text {eq }}$ equal to zero. If we assume that the time scale for the change of $m$ in the active layer is much larger than $T_{1, \mathrm{~s}}$ due to the reservoir provided by the cell, then $\partial m / \partial t \ll m / T_{1, \mathrm{~s}}$ and Eq. (A3) becomes

$$
\frac{\partial m}{\partial x}=-\frac{l_{\mathrm{a}} m}{D T_{1, \mathrm{~s}}} \quad \text { at } x= \pm L .
$$

Now the magnetization in the cell obeys the diffusion equation:

$$
\frac{\partial m}{\partial t}=\frac{-m}{T_{\text {in }}}+D \frac{\partial^{2} m}{\partial x^{2}},
$$

with the boundary condition Eq. (A4), while symmetry requires that

$$
\frac{\partial m}{\partial x}=0 \quad \text { at } \quad x=0
$$

We look for solutions of the form

$$
m_{q}(x, t)=\left(A_{q} \cos q x\right) e^{-p t},
$$

where the coefficients $A_{q}$ are determined by the condition that the initial magnetization $m(x, t=0)$ is given by the sum over all $q$. Substitution of $m_{q}(x, t)$ in Eq. (A5) yields

$$
D q^{2}=p-\frac{1}{T_{\text {in }}} .
$$

This equation couples to each mode $q$ a relaxation rate $p$, and it can directly be seen that the surface relaxation rate (diffusion inclusive) is $D q^{2}$. This means that the relaxation rate in the whole cell due to relaxation at the surface is $D q^{2}$, which is hence called the effective surface relaxation rate. The relaxation time of a mode $q$ decreases with $q^{2}$. As $m$ is a sum over all modes, the early recovery of $m$ will be nonexponential, but after the higher modes have decayed, $m$ will exponentially decay. Without intrinsic relaxation, $\left(T_{\mathrm{in}} \rightarrow \infty\right)$, there is only surface relaxation, and $p$ in Eq. (A7) then equals $D q^{2}$.

From the boundary condition [Eq. (A4)] we get the equation for mode selection

$$
q \tan q L=\frac{l_{\mathrm{a}}}{D T_{1, \mathrm{~s}}} .
$$

There are two limiting cases. If $l_{\mathrm{a}} / q T_{1, \mathrm{~s}} \ll D$, we are in the surface-limited regime, and the magnetization is homogeneous throughout the sample. From Eq. (A9) we get

$$
D q^{2}=\frac{l_{\mathrm{a}}}{L T_{1, \mathrm{~s}}},
$$

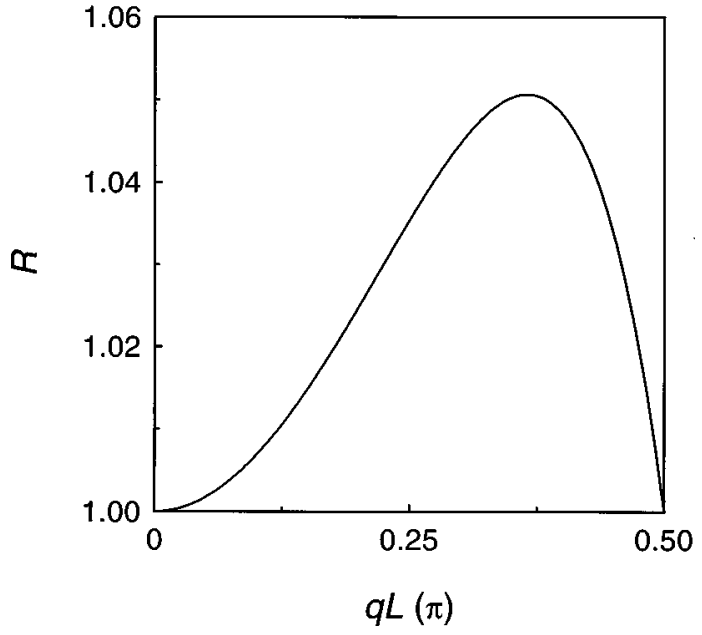

FIG. 11. Ratio $R$ of $T_{\mathrm{s}}\left[T_{\mathrm{s}}=\tau_{D}+T_{1, \mathrm{~s}}\left(L / l_{\mathrm{a}}\right)\right]$ to $1 / D q^{2}$, as a function of $q L$. The parameter varies from $q L=0$ (corresponding to surface-limited relaxation) to $q L=\pi / 2$ (corresponding to diffusionlimited relaxation).

which is, as expected, equal to the surface relaxation rate times $l_{\mathrm{a}} / L$, the ratio of the volumes of the active layer and the cell. We will henceforth denote $T_{1, \mathrm{~s}} L / l_{\mathrm{a}}$ the homogeneous relaxation time $T_{\text {hom }} \cdot q L \ll 1$ is equivalent to $D q^{2}$ $=1 / T_{\text {hom }} \ll D / L^{2}$, i.e., this regime applies if the diffusion time across the cell is much smaller than homogeneous relaxation time.

In the opposite limit, we are in the diffusion-limited regime, and we get from Eq. (A9)

$$
q L=\frac{\pi}{2}
$$

Hence the diffusion-limited relaxation rate becomes

$$
D q^{2}=D\left(\frac{\pi}{2 L}\right)^{2}=\frac{1}{\tau_{D}},
$$

which is equal to the inverse of the diffusion time $\tau_{D}$ for the lowest mode in a one-dimensional system of width $2 L$.

Let us define $T_{\mathrm{s}}$ as the sum of the diffusion time $\tau_{D}$ and the homogeneous relaxation time $T_{\text {hom }}$ :

$$
T_{\mathrm{s}} \equiv \tau_{D}+T_{\mathrm{hom}} \equiv \tau_{D}+T_{1, \mathrm{~s}} \frac{L}{l_{\mathrm{a}}},
$$

and calculate the $R$, the ratio of $T_{\mathrm{s}}$ to the real surface relaxation time $1 / D q^{2}$ :

$$
R=D q^{2} T_{\mathrm{s}}=D q^{2}\left(\tau_{D}+\frac{T_{1, \mathrm{~s}} L}{l_{\mathrm{a}}}\right) .
$$

With Eqs. (A9) and (A12), this can be written as

$$
R=\left(\frac{2 q L}{\pi}\right)^{2}+\frac{q L}{\tan q L} .
$$

$R$ is drawn in Fig. 11 as a function of $q L$, varying from $q L=0$, corresponding to surface-limited relaxation, to $q L$ $=\pi / 2$, corresponding to the diffusion-limited relaxation. It can be seen that the effective surface relaxation time $1 / D q^{2}$ can within $5 \%$ be approximated by $T_{\mathrm{s}}$. 
${ }^{1}$ R. L. Garwin and H. A. Reich, Phys. Rev. 115, 1478 (1959).

${ }^{2}$ F. J. Low and H. E. Rorschach, Phys. Rev. 120, 111 (1960); B. T. Beal and J. Hatton, ibid. 139, 1752 (1965); S. A. J. Wiegers, C. C. Kranenburg, T. Hata, R. Jochemsen, and G. Frossati, Europhys. Lett. 10, 477 (1989).

${ }^{3}$ H. Godfrin, G. Frossati, B. Hebral, and D. Thoulouze, J. Phys. (Paris), Colloq. 41, C7-275 (1980).

${ }^{4}$ M. Lowe, P. C. Hammel, R. E. Ecke, K. S. Bedell, and M. Takigawa, Phys. Rev. B 37, 2281 (1988).

${ }^{5}$ D. Vollhardt and P. Wölfle, Phys. Rev. Lett. 47, 190 (1981).

${ }^{6}$ K. S. Bedell and D. E. Melzer, Phys. Lett. 106A, 312 (1984); K. S. Bedell and D. E. Melzer, J. Low Temp. Phys. 63, 215 (1986).

${ }^{7}$ S. M. Havens-Sacco and A. Widom, Phys. Rev. B 25, 6696 (1982).

${ }^{8}$ A. S. van Steenbergen, S. A. J. Wiegers, J. A. A. J. Perenboom, and J. C. Maan, Phys. Rev. Lett. 79, 115 (1997).

${ }^{9}$ P. C. Hammel and R. C. Richardson, Phys. Rev. Lett. 52, 1441 (1984); R. C. Richardson, Physica B 126, 298 (1984); A. Schuhl, S. Maegawa, M. W. Meisel, and M. Chapellier, Phys. Rev. B 36, 6811 (1987).

${ }^{10}$ L. P. Roobol, S. C. Steel, R. Jochemsen, G. Frossati, K. S. Bedell, and A. E. Meyerovich, Europhys. Lett. 17, 219 (1992).

${ }^{11}$ P. J. Nacher, I. Shinkoda, P. Schleger, and W. N. Hardy, Phys. Rev. Lett. 67, 839 (1991); D. Candela, M. E. Hayden, and P. J. Nacher, ibid. 73, 2587 (1994); A. Rodrigues and G. Vermeulen, J. Low Temp. Phys. 108, 103 (1997).

${ }^{12}$ N. Bloembergen, E. M. Purcell, and R. V. Pound, Phys. Rev. 73, 679 (1948); A. Abragam, Principles of Nuclear Magnetism (Clarendon, Oxford, 1961).

${ }^{13}$ Hysol Epoxy CP4-4285, The Dexter Corporation, Olean, New York.

${ }^{14}$ A. S. van Steenbergen, S. A. J. Wiegers, J. A. A. J. Perenboom, and J. C. Maan, Physica B 211, 316 (1995).
${ }^{15}$ J. A. A. J. Perenboom, K. van Hulst, S. A. J. Wiegers, and J. C. Maan, Physica B 201, 507 (1994).

${ }^{16}$ Kapton, polyimide film, DuPont, Geneva, Switzerland.

${ }^{17} 1.001 \mathrm{k} \Omega$ resistor CRCW-0805, Dale Electronics, Columbus, Nebraska.

${ }^{18}$ Germanium temperature sensor GR-200A-30, Lakeshore, Westerville, Ohio.

${ }^{19}$ J. P. Eisenstein, Appl. Phys. Lett. 46, 695 (1985).

${ }^{20}$ E. D. Nelson and W. J. Mullin, Physica B 194-196, 889 (1994).

${ }^{21}$ V. P. Peshkov, JETP Lett. 21, 162 (1975).

${ }^{22}$ N. Alikacem, D. T. Sprague, and R. B. Hallock, Phys. Rev. Lett. 67, 2501 (1991).

${ }^{23}$ H. R. Hart and J. C. Wheatley, Phys. Rev. Lett. 4, 1 (1960); A. C. Anderson, W. Reese, R. J. Sarwinski, and J. C. Wheatley, ibid. 7, 220 (1961).

${ }^{24}$ A. C. Anderson, D. O. Edwards, W. R. Roach, R. E. Sarwinski, and J. C. Wheatley, Phys. Rev. Lett. 17, 367 (1966).

${ }^{25}$ See, e.g., J. Wilks and D. S. Betts, An Introduction to Liquid Helium, 2nd ed. (Clarendon, Oxford, 1987).

${ }^{26}$ V. Lefèvre-Seguin, P. J. Nacher, J. Brossel, F. Laloë, and W. N. Hardy, J. Phys. (Paris) 46, 1145 (1985).

${ }^{27}$ Y. Kondo, Y. Kodama, Y. Hirayoshi, T. Mizusaki, A. Hirai, and K. Eguchi, Phys. Lett. A 123, 417 (1987).

${ }^{28}$ D. S. Greywall, Phys. Rev. B 27, 2747 (1983).

${ }^{29}$ R. H. Romer, Phys. Rev. 117, 1183 (1960).

${ }^{30} \mathrm{D}$. Vollhardt (private communication).

${ }^{31}$ W. J. Mullin, F. Laloë, and M. G. Richards, J. Low Temp. Phys. 80, 1 (1990).

${ }^{32}$ B. Schizgal, J. Chem. Phys. 58, 3424 (1973).

${ }^{33}$ R. Chapman and M. G. Richards, Phys. Rev. Lett. 33, 18 (1974).

${ }^{34}$ R. Chapman, Phys. Rev. A 12, 2333 (1975).

${ }^{35}$ C. P. Lusher, M. F. Secca, and M. G. Richards, J. Low Temp. Phys. 72, 25 (1988); 72, 71 (1988). 